\title{
Metabolomic profiling of female mink serum during early to mid-pregnancy to understand factors associated with embryo diapause
}

zheng li

Jilin University

chengjun liu

Jilin University

liang deng

Jilin University

maosheng cao

Jilin University

xue zheng

Jilin University

lu chen

Jilin University

chenfeng yuan

Jilin University

zijiao zhao

Jilin University

chunjin li ( $\square$ llcjj158@163.com )

Research

Keywords: Metabolomic profiling, embryo diapause, Mink, Reactivation, Amino 12 acid, Dopamine

Posted Date: May 6th, 2020

DOI: https://doi.org/10.21203/rs.3.rs-23706/v1

License: (c) (1) This work is licensed under a Creative Commons Attribution 4.0 International License.

Read Full License 
1 Metabolomic profiling of female mink serum during early to

2 mid-pregnancy to understand factors associated with

3 embryo diapause

4 Zheng Li ${ }^{1}$, Chengjun Liu ${ }^{1}$, Liang Deng ${ }^{1}$, Maosheng Cao ${ }^{1}$, Xue Zheng ${ }^{1}$,Lu

5 Chen ${ }^{1}$,Chenfeng Yuan ${ }^{1}$, Zijiao Zhao ${ }^{1}$, Chunjin $\mathbf{L i}^{2 *}$

$6 \quad{ }^{1}$ College of Animal Sciences, Jilin University, Changchun, China.

$7 \quad{ }^{2}$ College of Animal Sciences, Jilin University, Changchun, China

8 * Correspondence:

9 Professor Chunjin Li

10 1lcjj158@163.com

11 Keywords: Metabolomic profiling; Embryo diapause; Mink; Reactivation; Amino

12 acid; Dopamine

\section{Highlights:}

We examined changes in the chemical composition of the serum extracts taken during the different stages of pregnancy.

Dopamine may be the major inhibitory compound for mink embryo reactivation

The levels of L-proline, L-threonine, taurine, D-ornithine, L-valine, L-kynurenine, and particularly L-leucine may be related to embryo reactivation.

\section{Abstract}

Background: The mink exhibits embryo diapause after fertilization.Mink embryos enter a period of diapause after the embryo develops into the blastocyst. The specific process of embryo diapause regulation and reactivation is remains largely un-examined. The aim of this study was to identify key factors associated with mink embryo diapause and reactivation by comparing and analyzing differences in serum metabolites up to twenty-nine days after mating.

Material and methods: Blood samples were taken on the first day of mating, and then once a week until the fifth week. Metabolomic profiles of the serum samples taken during this period were analysed by ultra-performance liquid chromatography/mass spectrometry. 
Results: Multivariate statistical analyses identified differential metabolite expression at different time points in both positive ion mode and negative ion modes. Dopamine may be the major inhibitory compound for mink embryo reactivation; moreover, and the levels of L-proline, L-threonine, taurine, D-ornithine, L-valine, L-kynurenine, and particularly L-leucine may be related to embryo reactivation.

Conclusions: We compared blood serum metabolites at different stages in the pregnancy. The study makes a significant contribution to the literature as we found that dopamine is a strong candidate as an inhibitor of embryo reactivation. The study showed that levels of seven amino acids, but especially L-leucine, may be correlated with embryo reactivation.

\section{Introduction}

The American mink (Neovison vison) exhibits seasonal estrus and mating-stimulated ovulation. When the fertilized oocyte develops into a blastocyst, the embryo is discharged from the uterus and enters a relatively static developmental state, called embryo diapause[1]. Birth of the offspring is postponed by delayed implantation of blastocysts so that offspring are born during favorable environmental conditions of temperature and food availability. This reproductive strategy increases the survival rate of the offspring.

From the vernal equinox, the photoperiod begins to increases gradually, which stimulates the secretion of prolactin from the pituitary gland[2] and initiates the development of the corpus luteum, which, in turn, promotes an increase in progesterone $\left(\mathrm{P}_{4}\right)$ secretion[3, 4]. When the level of $\mathrm{P}_{4}$ in the body reaches the requirement for blastocyst attachment to the uterine wall, the blastocysts begin to implant and enter the fetal developmental period[4]. Since the embryos maintain only basic metabolism during diapause, there is almost no mitosis or protein synthesis. The embryo is thus in a free state within the uterus[3].

The uterus is the most important environment for embryonic development and diapause, so the uterus and intrauterine substances play an important role in the diapause and in reactivating of the embryo. In a recent study, the effects of polyamines on diapaused mink embryos was validated. Treatment of mink uterine epithelial cells with different doses of prolactin revealed that this hormone induces the expression of polyamine regulatory genes such as $O D C 1$ in the uterus through the pSTAT1 and mTOR pathways, thereby regulating the levels of polyamine in the uterus[5]. In order to determine whether polyamines can alleviate embryo diapause, embryos in diapause were incubated in vitro with putrescine, resulting in an increase in blastocyst volume and the total number of cells, as indicated by further development of embryos; non-treated control embryos, however, remained in a diapause state[5]. Together, these results provide strong evidence showing that embryos are maintained in a diapause state because of a restricted supply of uterine-derived polyamines. 
Differential gene expression has been observed between mink embryos in diapause and reactivation mode. Over 200 embryonic genes were up-regulated during activation. Expression of embryonic genes related to polyamine synthesis increased during reactivation. Of the sequences corresponding to the genes characterized, about $14 \%$ were related to the cell cycle, and $14 \%$ were related to metabolism[5]; therefore, it can be inferred that metabolism may play an important role in the process of embryonic diapause and reactivation. Furthermore, as far as we know, no studies have investigated the changes in the metabolome of minks before and after embryo diapause.

Metabolomics an important component of systems biology. It is the science that studies the interactions between endogenous metabolites and internal or external factors in whole organisms, systems, organs, and cells[6]. Metabolomic analysis techniques consist of gas or liquid chromatography coupled to mass spectrometry (GC-MS/LC-MS), infrared spectroscopy (IR), and nuclear magnetic resonance (NMR). Among these technologies, LC/MS is advantageous because of its high resolution, high sensitivity, and reproducibility. Consequently, it has been widely used for the identification and quantification of metabolites[7]. It is used to identify and quantify hundreds of metabolites with high mass accuracy for comprehensive metabolic profiling[8]. Here, we examined changes in the chemical composition of the serum extracts taken during the different stages of pregnancy. Dopamine and several amino acids can be used as markers for embryo diapause and reactivation. To the best of our knowledge, no study has analyzed the metabolic changes taking place from fertilization to embryo diapause, from embryo diapause to embryo reactivation, during development, and during implantation. Our research is intended to support scientific progress in this important field.

\section{Materials and Methods}

\subsection{Animals and serum collection}

All experiments on minks were guided by and with the approval of the Animal Protection and Utilization Committee of Jilin University. Twenty multiparity minks were farmed in the Special Economic Animal Experiment Base of the Chinese Academy of Agricultural Sciences in Changchun (Jilin Province, P.R. China). All minks were raised under conditions of controlled temperature $\left(22-24^{\circ} \mathrm{C}\right)$ and humidity $(60-70 \%)$, where they were provided with sufficient food and water. Each female mink was mated with a fertile male according to usual practice. Venous blood from the 20 female minks was taken one day (D1), eight days (D8), 15 days (D15), 22 days (D22), and 29 days (D29) after mating. The blood was marked and transported at $2-8^{\circ} \mathrm{C}$ to the laboratory, centrifuged at $15,000 \mathrm{x}$ g for $15 \mathrm{~min}$, and the serum was stored at $-80^{\circ} \mathrm{C}$ until analysis. The 20 minks were fed and observed continually after blood collection until the end of pregnancy. Of the 20 minks used, 15 subsequently gave birth. Serum samples from six minks that shared the same gestation period (41 days) were ultimately used for metabolomic analysis. Six minks sera as three 
biological replicates. The two sera of each sampling day were mixed for metabolomic profiling.

\subsection{Sample preparation}

A serum sample $(100 \mu \mathrm{L})$ was placed in an EP tube, to which $300 \mu \mathrm{L}$ of methanol and $20 \mu \mathrm{L}$ internal standard substances were added, followed by vortexing for $30 \mathrm{sec}$. The samples were treated with ultrasound for $10 \mathrm{~min}$ (while incubated in ice water) and then incubated for $1 \mathrm{~h}$ at $-20^{\circ} \mathrm{C}$ to precipitate proteins. The sample was then centrifuged at $15,000 \mathrm{x}$ g for $15 \mathrm{~min}$ at $4^{\circ} \mathrm{C}$. The supernatant $(200 \mu \mathrm{L})$ was transferred into a fresh $2 \mathrm{~mL}$ LC/MS glass vial, $20 \mu \mathrm{L}$ were taken from each sample and pooled together as QC samples. An additional $200 \mu \mathrm{L}$ of supernatant were taken for UHPLC-QTOF-MS analysis.

\subsection{LC/MS analysis}

Metabolomic analysis was performed using an Agilent 1290 ultra-performance LC system coupled to quadrupole time-of-flight (Triple TOF 5600 AB Sciex) mass spectrometer, which is capable of performing primary and secondary mass spectrometry data acquisition based on the IDA function under the control of Analyst TF 1.7 software (AB Sciex). A UPLC BEH Amide column from Waters (1.7 $\mu \mathrm{m} * 2.1 * 100 \mathrm{~mm}$ ) was used for chromatographic separation; the column temperature was maintained at $40^{\circ} \mathrm{C}$. The LC-MS system was run in a binary gradient solvent mode. Solvent A contained $25 \mathrm{mM}$ ammonium acetate and $25 \mathrm{mM}$ ammonium hydroxide in water $(\mathrm{pH} 9.75)$, and solvent $\mathrm{B}$ contained $0.1 \%$ formic acid in acetonitrile. The flow rate was $500 \mu \mathrm{L} / \mathrm{min}$. The linear gradient was as follows: $0 \mathrm{~min}$, 95\% B; $7 \mathrm{~min}, 65 \% \mathrm{~B} ; 9 \mathrm{~min}, 40 \% \mathrm{~B} ; 9.1 \mathrm{~min}, 95 \% \mathrm{~B} ; 12 \mathrm{~min}, 95 \% \mathrm{~B}$ (Table 1). Sample analysis was performed using the positive or negative electrospray ionization (ESI) mode; the injection volume was $3 \mu \mathrm{L}$. In each cycle, twelve precursor ions with an intensity greater than 100 were chosen for fragmentation at a collision energy (CE) of $30 \mathrm{~V}$ (15 MS/MS events with a production accumulation time of $50 \mathrm{~ms}$ each). The ESI ion source parameters were set as follows: ion source gas 1 at 60 psi, ion source gas 2 at $60 \mathrm{psi}$, curtain gas at $35 \mathrm{psi}$, source temperature $650^{\circ} \mathrm{C}$, ion spray voltage floating (ISVF) $5,000 \mathrm{~V}$ or $-4000 \mathrm{~V}$ in positive or negative modes, respectively.

Quality control (QC) samples, which were prepared by mixing equal volumes $(20 \mu \mathrm{L})$ from each serum sample as they were aliquoted for analysis, were used to assess the reproducibility and reliability of the LC-MS system.

\subsection{Data processing and pattern recognition}

Unprocessed mass spectrometry data were converted to mzXML using ProteoWizard software and then processed with the R package XCMS v3.2.). The pre-processing results generated a data matrix that consisted of the retention time (RT), mass-to-charge ratio $(\mathrm{m} / \mathrm{z})$ values, and peak intensity. The $\mathrm{R}$ package CAMERA was used for peak annotation after XCMS data processing. 
The resulting scaled datasets were applied to the principal component analysis (PCA), which was used to validate the quality of the analytical system performance and to observe possible outliers. The orthogonal projections to latent structures-discriminant analysis (OPLS-DA) was used to analyse the results by SIMCA-P v11.0 software (Umetrics AB, Umea, Sweden) to filter out orthogonal variables in metabolites that are not related to categorical variables, and to analyse non-orthogonal variables and orthogonal variables separately to obtain an overview of the complete data set, and to discriminate between variables that are responsible for variation between the groups. OPLS-DA score plots were used to evaluate the quality of the model by the relevant R2 and Q2 parameters. The differential metabolites were selected by the combination of the P-value of the ANOVA and variable importance in the projection (VIP) values of the OPLS-DA model. The $P$-value was less than 0.05 , and the VIP value was larger than 1 . The $\log 2$-fold change was used to show how these selected differential metabolites varied between groups. Metabolites that varied were also shown by Volcano Plot. The online database KEGG (http://www.genome.jp/kegg/) was used to annotate the potential differential metabolites by searching for the exact molecular mass data from redundant $\mathrm{m} / \mathrm{z}$ peaks against a specific metabolite. Metabolomic analyses were performed by SPSS v22.0 software.

\section{Results}

\subsection{Original chromatogram based on LC /MS}

All sample analyses were performed in positive and negative electrospray ionization (ESI) mode by using ultra-performance LC quadrupole time-of-flight tandem MS (UPLCQ-TOF-MS). A total of 849 metabolites were detected in positive ion mode, 853 metabolites in negative ion mode (Supplement 1). A broad overlapping spectrum of QC samples showed that the instrument has repeatability and stability (Fig. 1).

\subsection{Multivariate statistical analysis}

PCA and orthogonal partial least-squares discriminant analysis (OPLS-DA) was performed on the data from the 15 samples (five stages $\times$ three biological replicates) to observe differences in metabolic profiles among different time points. The analysis showed that there was a clear separation between D1 and another group in the PD1 $\times$ PD8 score plots (Fig. 2). To further search for ion peaks that discriminated between the two groups, a supervised OPLS-DA model was established because it focused on actual class discrimination more than the unsupervised PCA model. As shown in Fig. 3, all R2X were greater than 0.5, R2Y was 1 or 0.999 , and Q2 was greater than 0.9 , except D1 vs D8 in positive ion mode, indicating that the OPLS-DA model had high predictive ability. The OPLS-DA models discriminated adequately between different groups, both in positive and negative ion modes, which indicated that their metabolic characteristics were distinct. To validate the reliability of the OPLS-DA model, an alignment verification was performed (Fig. 4). R2' and Q2' were lower than R2 and Q2 of the original model, which implies that the corresponding 
points did not exceed the corresponding lines, indicating that the model was meaningful. Thus, the differential metabolites can be screened according to the VIP.

\subsection{Identification of Potential Biomarkers and Metabolic Pathways}

In the first instance, metabolites with a VIP value greater than 1 in the OPLS-DA model were selected. The ANOVA was used to select metabolites with significant changes $(P<0.05)$. A volcano plot (Fig. 5) revealed differences in expression levels of the metabolites in the two groups and the metabolites with statistically significant differences. A total of 33 and 49 differential metabolites of the comparison group 1 (D1 VS D8) could be annotated in positive mode and negative mode using the KEGG pathway database (Supplement 2). Most of the differentially-expressed amino acids annotated were down-regulated, including L-proline, L-threonine, taurine, L-leucine, D-ornithine, L-lysine, L-valine, L-kynurenine, and L-Glutamine. On the contrary, L-methionine, L-phenylalanine, and L-tyrosine were up-regulated in D8 as compared to D1. Similarly, dopamine and its precursor, tyramine, were significantly increased in D8 as compared to D1 (Table 2). According to the KEGG pathway database, 38 metabolites were annotated (Supplement 3 ) in positive mode and 61 in negative mode as differential metabolites of group 2 (D1 VS D15), more than half of which were the same for group 1 (D1 VS D8). Levels of tyrosine and dopamine in D8 and D15 were significantly higher than in D1, which is involved with the prolactin signaling pathway. Almost the types of amino acids that up-regulated or down-regulated are the same when compared to D8 and D15 with D1. Regarding group 3 (D1 VS D22), 45 differential metabolites in positive mode and 50 in negative mode were annotated (Supplement 4). As for group 4 (D1VS D29), 31 and 38 differential metabolites, respectively, in the positive and negative ion modes were members of metabolic pathways (Supplement 5). All amino acids were down-regulated on D22 as compared to D1, except L-methionine and L-phenylalanine, and L-phenylalanine on D29. Prolactin can induce polyamine synthesis, which is essential for embryo reactivation [9]; moreover, dopamine is the primary physiological inhibitor of prolactin secretion[10]. The higher the levels of dopamine on D8 and D15 indicated that the embryos were in diapause. The interval from implantation to parturition in mink lasts 28-30 days $[11,12]$. In our study, the intervals between the fourth blood sampling time (D22) to labor lasted less than 30 days for all six minks, which indicated that the embryos had already escaped from diapause and had implanted on D22, and it was mid-pregnancy on D29. Therefore, further comparative analysis was conducted for D8 vs D15 and D15 vs D22 with PCA and OPLS-DA. The data showed that there was a clear separation between D8 and D15, D15, and D22 in the PD1 $\times$ PD 8 score plots (Fig. 2S). For a more in-depth search for ion peaks that discriminated between the two groups, the supervised OPLS-DA model was established. As shown in Fig. 3S, the OPLS-DA model was highly predictive. To validate the reliability of the OPLS-DA model, an alignment verification was performed (Fig. 4S). R2' and Q2' were smaller than R2 and Q2 of the original model, indicating that the model was meaningful. Differential metabolites could be screened according to the VIP. Metabolites with a VIP value greater than 1 in the OPLS-DA model were selected, and the AVONA was 
used to select metabolites with significant changes $(P<0.05)$. Because many amino acids promote mouse embryo development and blastocyst activation[13, 14], we focused on the differential amino acids and dopamine in the different groups. Dopamine was significantly higher on D15 than on D8, and the tyramine and L-tyrosine, precursors of dopamine, were also higher on D15 than on D8, but L-glutamine and D-ornithine decreased. L-lysine, L-arginine, L-kynurenine, DL-phenylalanine, and L-phenylalanine were significantly upregulated on D15 compared to D8, while L-proline, taurine, and L-methionine increased slightly, but the difference was not significant (Table 3). Dopamine and its precursors, tyramine, and L-tyrosine decreased significantly on D22 as compared to D15, and most amino acids were also down-regulated, including L-tyrosine, L-proline, D-ornithine, L-lysine, L-leucine, L-tryptophan, DL-phenylalanine, and L-kynurenine; only L-glutamine and L-pyroglutamic acid increased significantly (Table 4).

\section{Conclusion}

This study investigates aspects of metabolism of the female American mink (Neovison vison). After fertilization, the mink blastocyst enters a stage of diapause before it is reactivated and enters into a developmental stage in the uterus. The reasons for this are probably related to availability of food sources and other environmental conditions. The specific processes controlling embryo diapause and reactivation have not previously been studied in the mink. Our aim was to identify key metabolites associated with embryonic diapause and reactivation. We compared blood serum metabolites at different stages in the pregnancy. The study makes a significant contribution to the literature as we found that dopamine is a strong candidate as an inhibitor of embryo reactivation. The study showed that levels of seven amino acids, but especially L-leucine, may be correlated with embryo reactivation.

\section{Discussion}

Dopamine is a precursor of noradrenaline and is involved in controlling fine movements and mental processes. Mink require mating to stimulate ovulation, and the fertilized eggs are formed 48-60 hours after mating. A fertilized egg takes about six days to form a blastocyst and reach the uterus. The embryo does not implant immediately but undergoes a period of cell cycle arrest[1]. As a result, oocyte maturation or fertilization occurs one day after mating D1), and the eighth day (D8) may be the embryo diapause period. Dopamine levels significantly increased in D8 as compared to D1, confirming that the blastocysts on D8 were in developmental arrest. This suggests that dopamine levels may be a biomarker of mink embryonic diapause. Similarly, dopamine concentration was higher on D15 than on D1, indicating that the embryos of D15 were also in diapause. Tyrosine and tyramine, precursors of dopamine, were also higher on D8 and D15 than on D1. It has been demonstrated that CYP2D6 can mediate the derivation of dopamine from tyramine, while tyrosine hydroxylase mediates the derivation of dopamine from tyrosine and that both tyrosine 
and tyramine can increase dopamine levels[15]. L-phenylalanine is a synthetic precursor of dopamine[16], which also impacts mouse embryo implantation negatively by disrupting cytokine-based immunity and oxidative stress in the uterus[17].The higher level of L-phenylalanine in the embryo of diapause stages D15 and D8 may have negative effects on polyamine synthesis and embryo implantation. We also found that dopamine and its precursors, L-phenylalanine, tyramine, and L-tyrosine, were increased on D15 as compared to D8, further indicating that the embryo diapause period was also occurring on D15. Dopamine and its precursors were lower on D22 than on D15, indicating that embryos on D22 had implanted, which was consistent with the suggestion that dopamine is a marker for mink embryo diapause.

In our study, The gestation period of minkis 41 days, we speculate that D15 may be the end of embryo diapause. Thus, increasing amino acid levels may be a precursor of embryo activation. Most amino acids increased to higher levels on D15 than on D8, notably L-kynurenine, L-lysine, and L-arginine, which are precursors of polyamine. After embryo implantation (D22), most amino acids decreased significantly as compared to D15 and D1, indicating that amino acids may play an important role in mink oocyte maturation and embryo reactivation. The levels of almost all amino acids were reduced to a greater extent after embryo implantation (D22) than at the end of the embryo diapause (D15), suggesting that the function of the amino acids may have already been completed, leaving no further need for high concentrations of amino acids after embryo reactivation.

As a major macronutrient, amino acids are not only a component of proteins and peptides, but they are also essential for the synthesis of many biologically active molecules involved in the regulation of signalling pathways and metabolism in the body. Experimental evidence demonstrates that amino acids play a key role in female and male reproduction[18, 19]. Many in vitro studies on mouse embryos have demonstrated that amino acids, especially essential amino acids, have a vital role in embryo development and blastocyst activation[9]. The embryonic development and implantation requirements of amino acids are not simply nutritive to support protein synthesis and trophoblast differentiation, but it also induces activation of mTOR-dependent signals after the embryo has reached the early blastocyst stage. The resultant signals cause transduction cascades, including triggering insulin-like growth factor II and polyamine synthesis in the blastocyst, and finally increasing cell motility[14, 20]. While polyamines are essential to folliculogenesis[21], fertilization[22], and early embryo development[23, 24], deficiency of polyamines causes embryo developmental arrest[9]. Most amino acids existed at greater levels on D1 than on D8, D15, D22, and D29 demonstrating that amino acids may induce an increase of polyamines via the mTOR pathway. Most amino acids annotated by the KEGG pathway database, including L-proline, L-threonine, taurine, L-leucine, D-ornithine, L-valine, and L-kynurenine, were down-regulated on D8 and D15 as compared to D1. Our study showed that while polyamines are scarce during embryo diapause[9], most amino acid levels were lower on D8 and D15 than on D1. $\mathrm{D}$-ornithine is a precursor of polyamine (putrescine), and arginine or proline can 
synthesize putrescine through ornithine by the highly-regulated rate-limiting enzyme, ornithine decarboxylase 1 (ODC1)[25].

Lower levels of D-ornithine and L-proline on D8 and D15 might cause a decrease in putrescine synthesis, thereby hindering embryonic reactivation and development. L-proline plays a novel role in the regulation of pluripotency and cell differentiation, which can induce embryonic stem cells (ESC) to form early primitive ectoderm-like cells[26]. In an in vitro mouse embryo culture, the omission of L-threonine significantly reduces mouse blastocyst hatching, attachment, and trophoblastic outgrowth[13]. L-threonine deprivation significantly decreases self-renewal markers and differentiation markers of embryonic stem cells[27]. The low concentrations of L-threonine on D8 and D15 coincided with the state of embryonic diapause. Taurine can improve embryonic development in vitro in several animals, including mouse[9], pig[28], cow[29], and rabbit[30]. What is more, taurine is an osmotic adjustment organic substance[31, 32]. Therefore, it is nearly impossible for taurine to spontaneously diffuse through the cell membrane because of its lyophobic properties, and thus an extremely high intracellular concentration may be maintained. Taurine acts as an osmolyte in human and mouse oocytes and embryos when they have to adjust their cell volume because of either extracellularly-induced or intracellularly-induced osmotic imbalances[33]. The low level of taurine on D8 and D15 may be involved with mink blastocysts diapause, which is of no benefit to blastocyst expansion and hatching. Human embryos that develop in vitro to the blastocyst stage in the presence of amino acids always accumulate leucine, but not other amino acids from the culture medium[34]. The decrease of leucine and valine levels may be related to the diapause of the mink blastocyst.

The diapause period usually lasts 15-25 days, but sometimes, it is shortened to a few days or extended up to 55 days[1, 35]. In hypophysectomised minks injected with prolactin, embryo implantation occurred on days 14 and 21 , but not at the same time[36]. In our study, the interval from embryo diapause to implantation occurred within seven days, indicating that a factor besides prolactin may be needed for the blastocysts to escape from diapause and begin development, or, possible, that other factors can accelerate embryo reactivation and implantation. The level of dopamine was elevated, and the level of leucine was decreased during the diapause period of the mink embryos. Changes in levels of dopamine and leucine may inhibit the production of prolactin. Taken together, changes to amino acid levels on D8, D15, and D22 indicated that amino acids, especially leucine, may also be another important factor in mink embryo diapause and reactivation. Leucine may have a synergistic effect with prolactin during the activation of mink embryos, and an increase in both the levels of leucine and prolactin may increase the rate of reactivation. Unfortunately, we did not collect blood from the beginning of embryo development to test this. In addition, these results need to be further verified by in vitro embryo culture and in vivo experiments. These issues should be further studied by subsequent experiments so that the mink embryo diapause and reactivation can be explored more comprehensively. 
To the best of our knowledge, this is the first study to investigate links between serum metabolites and mink embryo diapause. The level of dopamine int the serum may be used as a marker for embryonic diapause, and changes in the levels of amino acids may be associated with embryo reactivation. This study fills a gap in metabolome analysis in this field and provides some references for subsequent research.

6 Abbreviations

\begin{tabular}{|l|l|}
\hline \multicolumn{1}{|c|}{ Abbreviation } & \multicolumn{1}{c|}{ Meaning } \\
\hline P4 & progesterone \\
\hline GC-MS/LC-MS & $\begin{array}{l}\text { gas or liquid chromatography coupled to } \\
\text { mass spectrometry }\end{array}$ \\
\hline IR & infrared spectroscopy \\
\hline NMR & nuclear magnetic resonance \\
\hline UHPLC-QTOF-MS & $\begin{array}{l}\text { The ultra-high performance liquid } \\
\text { chromatography-quadrupole } \\
\text { time-of-flight mass spectrometry }\end{array}$ \\
\hline QC & Quality control \\
\hline ESI & negative electrospray ionization \\
\hline OPLS-DA & $\begin{array}{l}\text { ime-of-flight tandem MS } \\
\text { structures-discriminant analysis }\end{array}$ \\
\hline EPLCQ-TOF-MS & embryonic stem cells \\
\hline
\end{tabular}

\subsection{Ethical Approval and Consent to participate}


The study design was approved by the appropriate ethics review board. We have read and understood your journal's policies, and we believe that neither the manuscript nor the study violates any of these.

\subsection{Consent for publication}

All authors read and approved the final manuscript and, agree to the publication of the manuscript.

\subsection{Availability of supporting data}

All data, models, and code generated or used during the study appear in the submitted article.

\subsection{Conflict of Interest}

The authors declare that the research was conducted in the absence of any commercial or financial relationships that could be construed as a potential conflict of interest.

\subsection{Funding}

This research was supported by the National Key Research and Development Program of China (2017YFD0501701), The National Natural Science Foundation of China (31672417), and the Science and Technology Development Project of Changchun (17DY022).

\subsection{Author Contributions}

All of the authors contributed to the conception of the review. CJL designed the experiment, while ZL and LD performed the experiment. The first draft of the manuscript was prepared by CL. CJL performed subsequent amendments. ZL revised the manuscript. All authors read and approved the final manuscript.

\subsection{Acknowledgments}

We acknowledge the staff at the Special Economic Animal Experiment Base of the Chinese Academy of Agricultural Sciences for their help in providing the animals and collecting serum; as well as the staff at biomarker technologies for their excellent technical assistance.

\section{References}

1. Murphy BD, James DA: The effects of light and sympathetic innervation to the head on nidation in mink. Journal of Experimental Zoology 1974, 187:267-276.

2. Murphy B, DiGregorio G, Douglas D, Gonzalez-Reyna A: Interactions between melatonin and prolactin during gestation in mink (Mustela vison). Reproduction 1990, 89:423-429.

3. Papke RL, Concannon PW, Travis HF, Hansel W: Control of luteal function and implantation in the mink by prolactin. Journal of Animal Science 1980, 50:1102-1107. 
4. Martinet L, Allain D, Meunier M: Regulation in pregnant mink (Mustela vison) of plasma progesterone and prolactin concentrations and regulation of onset of the spring moult by daylight ratio and melatonin injections. Canadian journal of zoology 1983, 61:1959-1963.

5. Fenelon JC, Banerjee A, Lefèvre P, Gratian F, Murphy BD: Polyamine-mediated effects of prolactin dictate emergence from mink obligate embryonic diapause. Biology of reproduction 2016, 95:6, 1-13.

6. Tang H, Wang Y: Metabonomics: a revolution in progress. Sheng wu hua xue yu sheng wu wu li jin zhan 2006, 33:401-417.

7. Zhou B, Xiao JF, Tuli L, Ressom HW: LC-MS-based metabolomics. Molecular BioSystems 2012, 8:470-481.

8. Theodoridis G, Gika HG, Wilson ID: LC-MS-based methodology for global metabolite profiling in metabonomics/metabolomics. TrAC Trends in Analytical Chemistry 2008, 27:251-260.

9. ! !!! INVALID CITATION !!!

10. Massara F, Camanni F, Belforte L, Molinatti G: Dopamine and inhibition of prolactin and growth-hormone secretion. Lancet (London, England) 1976, 1:913-913.

11. Enders RK: Reproduction in the mink (Mustela vison). Proceedings of the American Philosophical Society 1952, 96:691-755.

12. Concannon P, Pilbeam T, Travis H: Advanced implantation in mink (Mustela vison) treated with medroxyprogesterone acetate during early embryonic diapause. Reproduction 1980, 58:1-6.

13. Spindle AI, Pedersen RA: Hatching, attachment, and outgrowth of mouse blastocysts in vitro: fixed nitrogen requirements. Journal of Experimental Zoology 1973, 186:305-318.

14. Martin PM, Sutherland AE: Exogenous amino acids regulate trophectoderm differentiation in the mouse blastocyst through an mTOR-dependent pathway. Developmental biology 2001, 240:182-193.

15. Zhu W, Mantione KJ, Shen L, Cadet P, Esch T, Goumon Y, Bianchi E, Sonetti $\mathrm{D}$, Stefano GB: Tyrosine and tyramine increase endogenous ganglionic morphine and dopamine levels in vitro and in vivo: cyp2d6 and tyrosine hydroxylase modulation demonstrates a dopamine coupling. Medical Science Monitor 2005, 11:BR397-BR404.

16. Kapatos G, Zigmond M: DOPAMINE BIOSYNTHESIS FROM LTYROSINE AND L-PHENYLALANINE IN RAT BRAIN SYNAPTOSOMES: PREFERENTIAL USE OF NEWLY ACCUMULATED PRECURSORS 1, 2. Journal of neurochemistry 1977, 28:1109-1119. 
17. Dong Y, Bai Y, Liu G, Wang Z, Cao J, Chen Y, Yang H: The immunologic and antioxidant effects of $L$-phenylalanine on the uterine implantation of mice embryos during early pregnancy. Histology and histopathology 2014, 29:1335-1342.

18. Wu G, Bazer FW, Dai Z, Li D, Wang J, Wu Z: Amino acid nutrition in animals: protein synthesis and beyond. Annu Rev Anim Biosci 2014, 2:387-417.

19. Bazer FW: Contributions of an animal scientist to understanding the biology of the uterus and pregnancy. Reproduction, Fertility and Development 2012, 25:129-147.

20. Martin PM, Sutherland AE, Van Winkle LJ: Amino acid transport regulates blastocyst implantation. Biology of reproduction 2003, 69:1101-1108.

21. Bastida CM, Cremades An, Castells MT, López-Contreras AsJ, López-García $\mathrm{C}$, Tejada F, Peñafiel R: Influence of ovarian ornithine decarboxylase in folliculogenesis and luteinization. Endocrinology 2005, 146:666-674.

22. Fleming AD, Armstrong DT: Effects of polyamines upon capacitation and fertilization in the guinea pig. Journal of Experimental Zoology 1985, 233:93-100.

23. Alexandre H: Effect of inhibitors of polyamine biosynthesis on primary differentiation of mouse egg. Comptes rendus hebdomadaires des seances de l'Academie des sciences Serie D: Sciences naturelles 1978, 286:1215-1217.

24. Part M, Prakash N, Grove J, Schechter P, Sjoerdsma A, Koch-Weser J: L-Ornithine decarboxylase: an essential role in early mammalian embryogenesis. Science 1980, 208:505-508.

25. Wallace HM, Fraser AV, Hughes A: A perspective of polyamine metabolism. Biochemical Journal 2003, 376:1-14.

26. Washington JM, Rathjen J, Felquer F, Lonic A, Bettess MD, Hamra N, Semendric L, Tan BSN, Lake J-A, Keough RA: L-Proline induces differentiation of ES cells: a novel role for an amino acid in the regulation of pluripotent cells in culture. American Journal of Physiology-Cell Physiology 2010, 298:C982-C992.

27. Wang J, Alexander P, Wu L, Hammer R, Cleaver O, McKnight SL: Dependence of mouse embryonic stem cells on threonine catabolism. Science 2009, 325:435-439.

28. Petters R, Wells K: Culture of pig embryos. Journal of reproduction and fertility Supplement 1993, 48:61-73.

29. Liu Z, Foote RH: Development of bovine embryos in KSOM with added superoxide dismutase and taurine and with five and twenty percent 02. Biology of reproduction 1995, 53:786-790. 
30. Li J, Foote RH, Simkin M: Development of rabbit zygotes cultured in protein-free medium with catalase, taurine, or superoxide dismutase. Biology of reproduction 1993, 49:33-37.

31. Huxtable R: Physiological actions of taurine. Physiological reviews 1992, 72:101-163.

32. McManus ML, Churchwell KB, Strange K: Regulation of cell volume in health and disease. New England Journal of Medicine 1995, 333:1260-1267.

33. Dumoulin JC, Wissen LCV, Menheere PP, Michiels AH, Geraedts JP, Evers JL: Taurine acts as an osmolyte in human and mouse oocytes and embryos. Biology of Reproduction 1997, 56:739.

34. Hemmings K, Maruthini D, Vyjayanthi S, Hogg J, Balen A, Campbell B, Leese $\mathrm{H}$, Picton $\mathrm{H}$ : Amino acid turnover by human oocytes is influenced by gamete developmental competence, patient characteristics and gonadotrophin treatment. Human Reproduction 2013, 28:1031-1044.

35. Martinet L, Allais C, Allain D: The role of prolactin and LH in luteal function and blastocyst growth in mink (Mustela vison). Journal of reproduction and fertility Supplement 1981, 29:119.

36. Murphy BD, Concannon PW, Travis HF, Hansel W: Prolactin: The Hypophyseal Factor That Terminates Embryonic Diapause in Mink. Biology of Reproduction 1981, 25:487.

Table 1 The gradient of the mobile phase.

\begin{tabular}{llll}
\hline $\begin{array}{l}\text { Time } \\
(\min )\end{array}$ & $\begin{array}{l}\text { Flow rate } \\
(\mu \mathrm{L} / \mathrm{min})\end{array}$ & $\mathrm{A} \%$ & $\mathrm{~B} \%$ \\
\hline 0 & 500 & 5 & 95 \\
0.5 & 500 & 5 & 95 \\
7 & 500 & 35 & 65 \\
8 & 500 & 60 & 40 \\
9 & 500 & 60 & 40 \\
9.1 & 500 & 5 & 95
\end{tabular}


Table 2 Amino acids and dopamine change (D1 VS D8)

\begin{tabular}{|c|c|c|c|c|c|}
\hline NO. & name & $\begin{array}{l}\log 2 \text { Fold } \\
\text { Change(D8/D1 } \\
\text { ) }\end{array}$ & P-value & VIP & regulated \\
\hline 1 & L-Proline & -0.599 & 0.003 & 1.300 & down \\
\hline 2 & L-Valine & -0.703 & 0.004 & 1.294 & down \\
\hline 3 & L-Threonine & -0.656 & 0.015 & 1.225 & down \\
\hline 4 & Taurine & -0.467 & 0.049 & 1.201 & down \\
\hline 5 & L-Leucine & -0.381 & 0.021 & 1.204 & down \\
\hline 6 & L-Methionine & 1.054 & 0.036 & 1.249 & up \\
\hline 7 & L-Phenylalanine & 1.033 & 0.001 & 1.358 & up \\
\hline 8 & L-Glutamine & -0.334 & 0.030 & 1.198 & down \\
\hline 9 & Tyramine & 0.982 & 0.022 & 1.474 & up \\
\hline 10 & Dopamine & 0.682 & 0.000 & 1.543 & up \\
\hline 11 & D-Ornithine & -1.213 & 0.004 & 1.487 & down \\
\hline 12 & L-Tyrosine & 0.916 & 0.000 & 1.535 & up \\
\hline 13 & L-Lysine & -0.576 & 0.002 & 1.518 & down \\
\hline 14 & L-Kynurenine & -0.551 & 0.000 & 1.533 & down \\
\hline
\end{tabular}


Table 3 Amino acids and dopamine change (D8 VS D15)

\begin{tabular}{|c|c|c|c|c|c|}
\hline NO. & name & $\begin{array}{l}\log 2 \text { Fold } \\
\text { Change(D15/D } \\
8)\end{array}$ & P-value & VIP & regulated \\
\hline 1 & L-Proline & 0.145 & 0.133 & 1.062 & unchanged \\
\hline 2 & Tyramine & 0.535 & 0.012 & 1.266 & up \\
\hline 3 & Taurine & 0.518 & 0.058 & 1.128 & unchanged \\
\hline 4 & Dopamine & 0.456 & 0.000 & 1.379 & up \\
\hline 5 & L-Methionine & 0.640 & 0.052 & 1.160 & unchanged \\
\hline 6 & L-Phenylalanine & 0.702 & 0.036 & 1.246 & up \\
\hline 7 & D-Ornithine & -1.058 & 0.032 & 1.282 & down \\
\hline 8 & L-Tyrosine & 0.347 & 0.002 & 1.364 & up \\
\hline 9 & L-Lysine & 0.956 & 0.007 & 1.343 & up \\
\hline 10 & L-Kynurenine & 0.397 & 0.018 & 1.283 & up \\
\hline 11 & DL-Phenylalanine & 0.681 & 0.029 & 1.189 & up \\
\hline 12 & L-Arginine & 0.175 & 0.045 & 1.215 & up \\
\hline 13 & L-Glutamine & -0.705 & 0.001 & 1.412 & down \\
\hline
\end{tabular}

Table 4 Amino acids and dopamine change (D15 VS D22)

\begin{tabular}{lllll}
\hline NO. name & $\begin{array}{l}\log 2 \text { Fold } \\
\text { Change(D22/D }\end{array}$ & P-value & VIP & regulated \\
\hline
\end{tabular}




\begin{tabular}{|c|c|c|c|c|c|}
\hline & & 15) & & & \\
\hline 1 & L-Leucine & -0.263 & 0.038 & 1.268 & down \\
\hline 2 & L-Glutamine & 0.512 & 0.010 & 1.374 & up \\
\hline 3 & L-Proline & -0.501 & 0.016 & 1.429 & down \\
\hline 4 & Tyramine & -0.353 & 0.035 & 1.370 & down \\
\hline 5 & D-Ornithine & -0.581 & 0.008 & 1.360 & down \\
\hline 6 & DL-Phenylalanine & -0.998 & 0.018 & 1.329 & down \\
\hline 7 & Dopamine & -0.993 & 0.008 & 1.464 & down \\
\hline 8 & L-Tyrosine & -0.961 & 0.033 & 1.402 & down \\
\hline 9 & L-Lysine & -0.556 & 0.023 & 1.387 & down \\
\hline 10 & L-Tryptophan & -0.535 & 0.023 & 1.325 & down \\
\hline 11 & L-Kynurenine & -0.891 & 0.002 & 1.449 & down \\
\hline 12 & $\begin{array}{l}\text { L-Pyroglutamic } \\
\text { acid }\end{array}$ & 0.768 & 0.029 & 1.309 & up \\
\hline
\end{tabular}

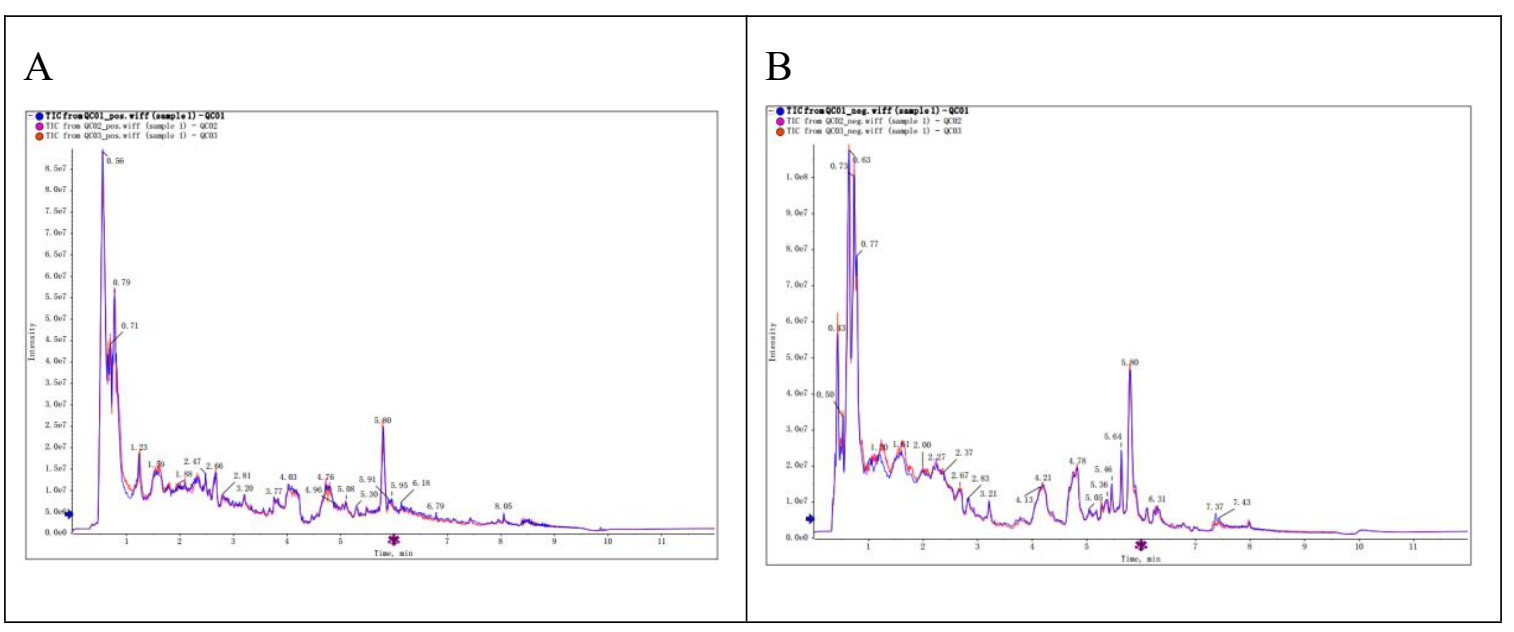

516 Fig. 1 Total Ion Chromatogram of QC in ESI+ mode (A) and ESI -mode (B) 


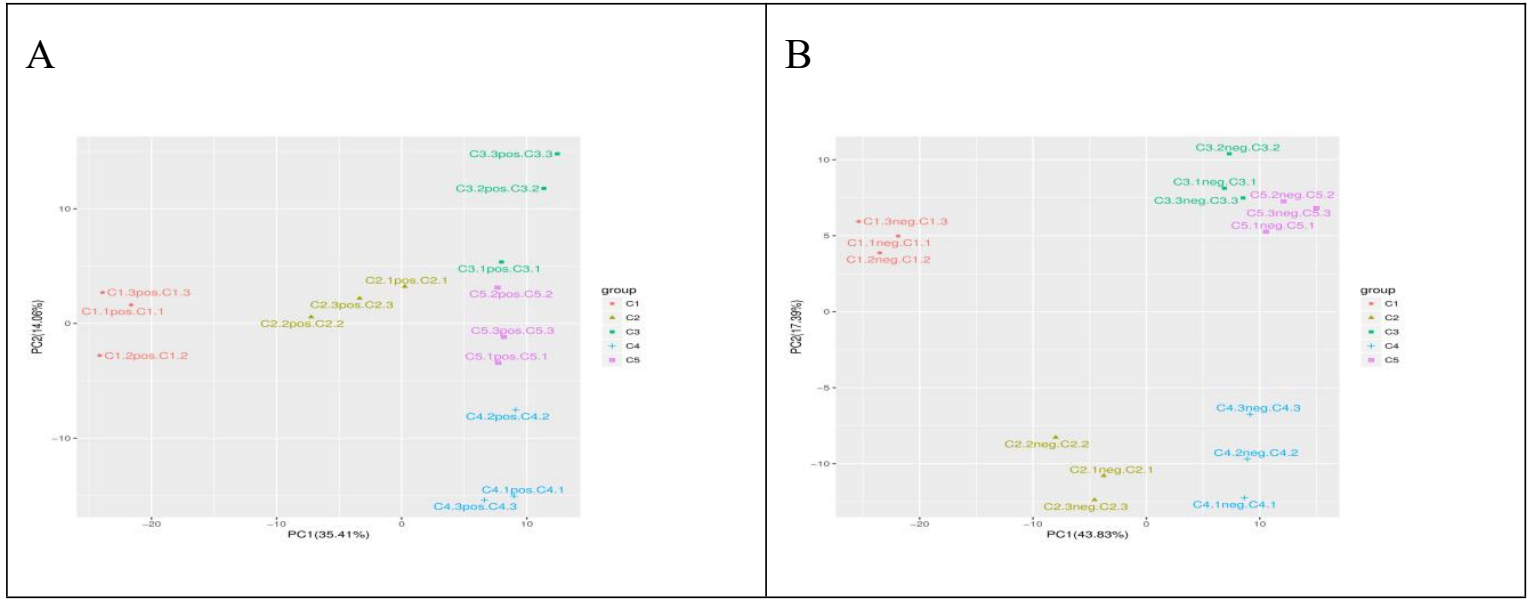

Fig. 2 PCA score plots in the five groups in ESI+ mode (A) and ESI- mode (B).

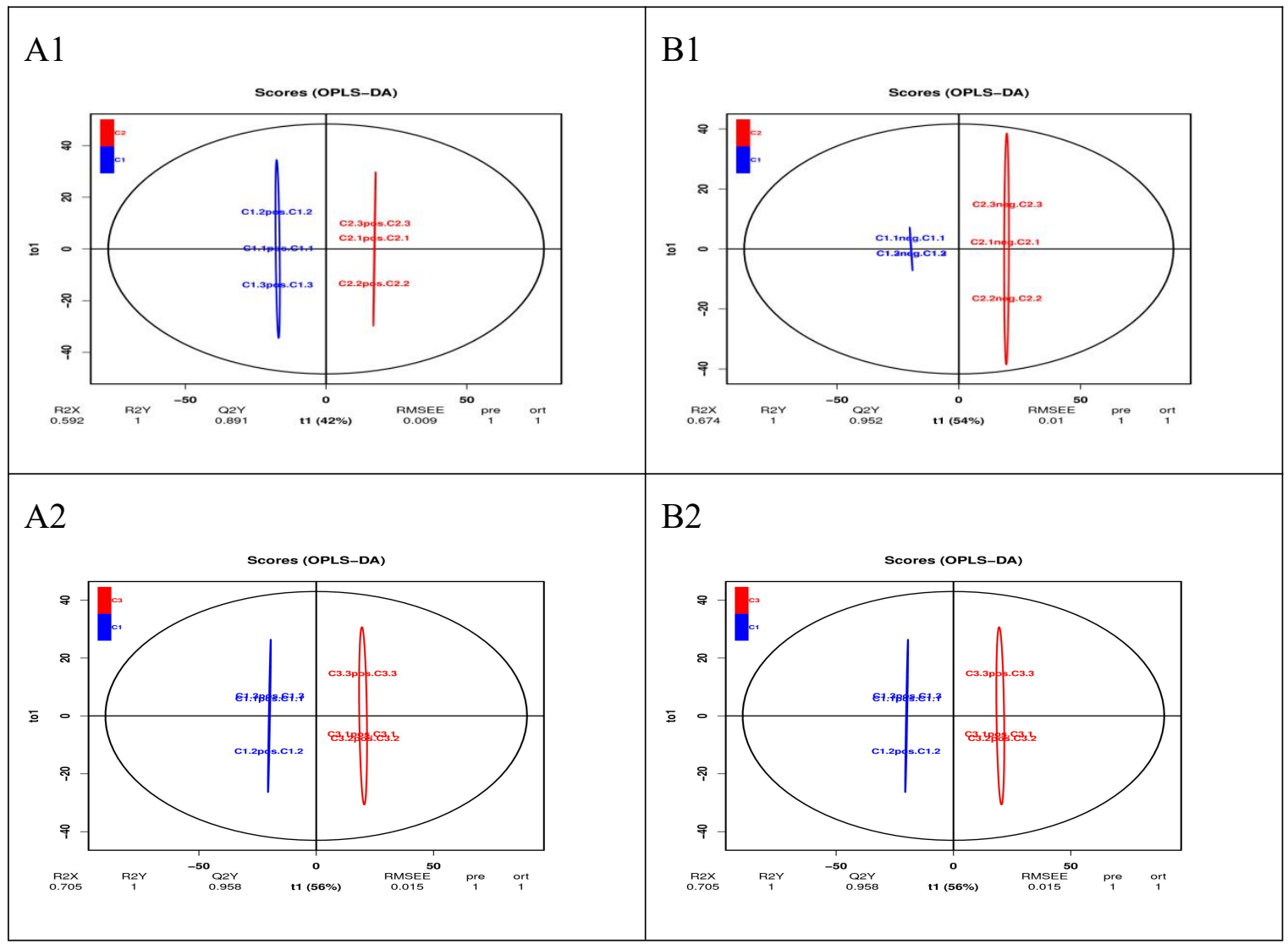




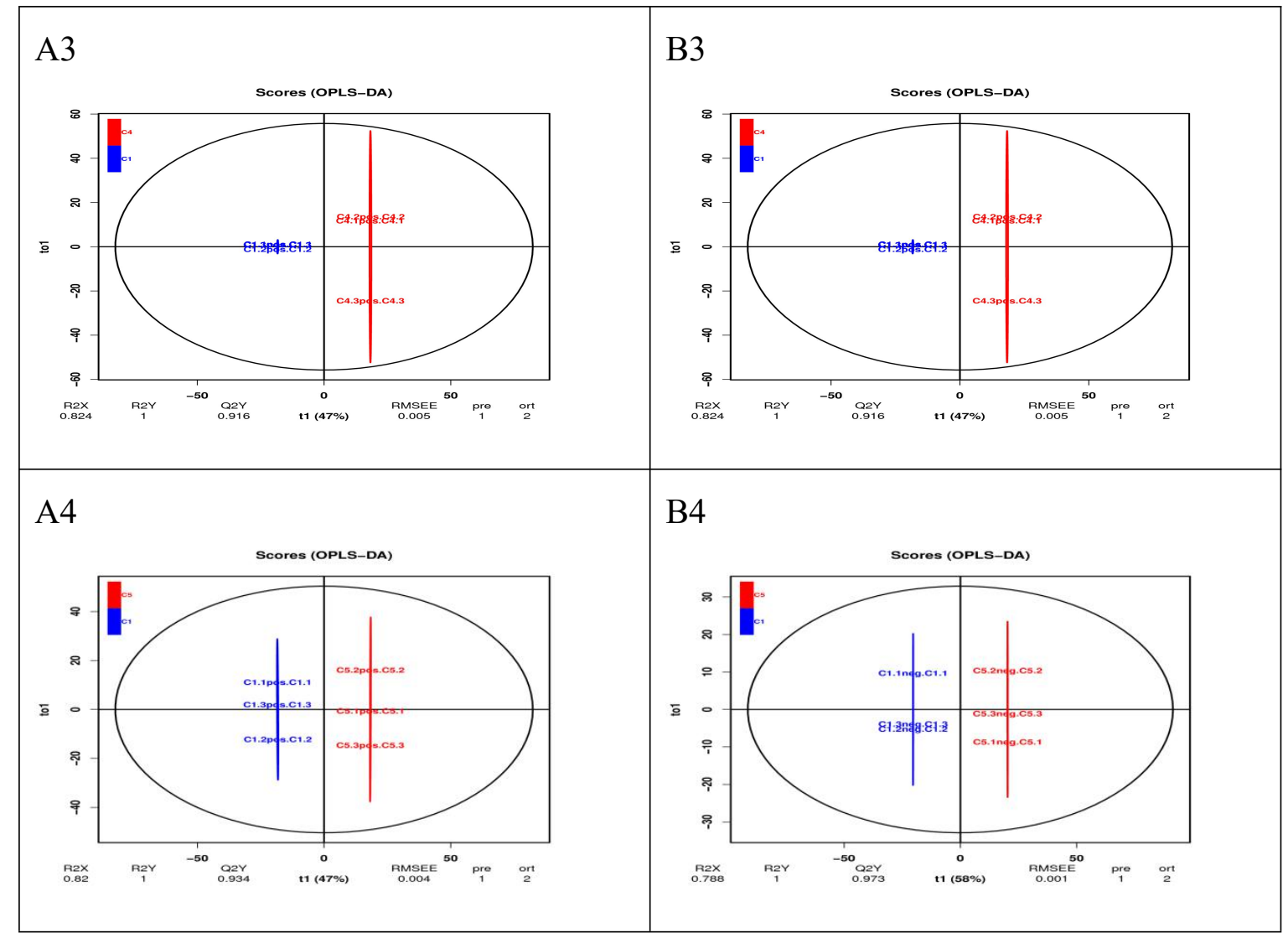

Fig. 3 OPLS-DA score plot of the four comparisons: (A1) D1 vs D8 in ESI+ mode, (B1) D1 vs D8 in ESI- mode; (A2) D1 vs D15 in ESI+ mode, (B2) D1 vs D15 in ESImode; (A3) D1 vs D22 in ESI+ mode, (B3) D1 vs D22 in ESI- mode; (A4) D1 vs D29 in ESI+ mode, (B4) D1 vs D29 in ESI- mode.

A1

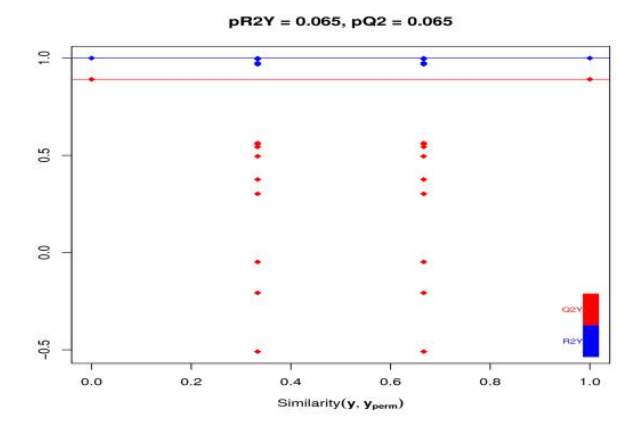

B1

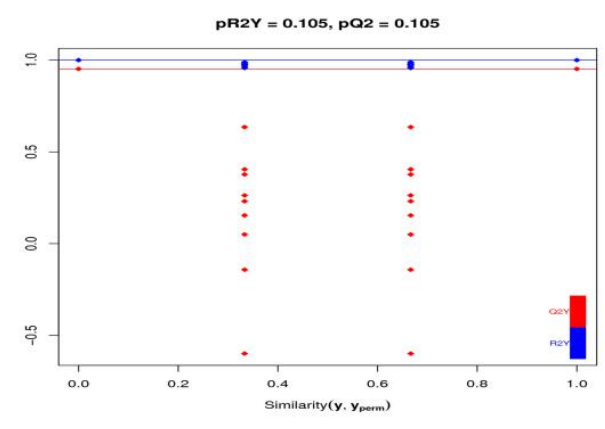




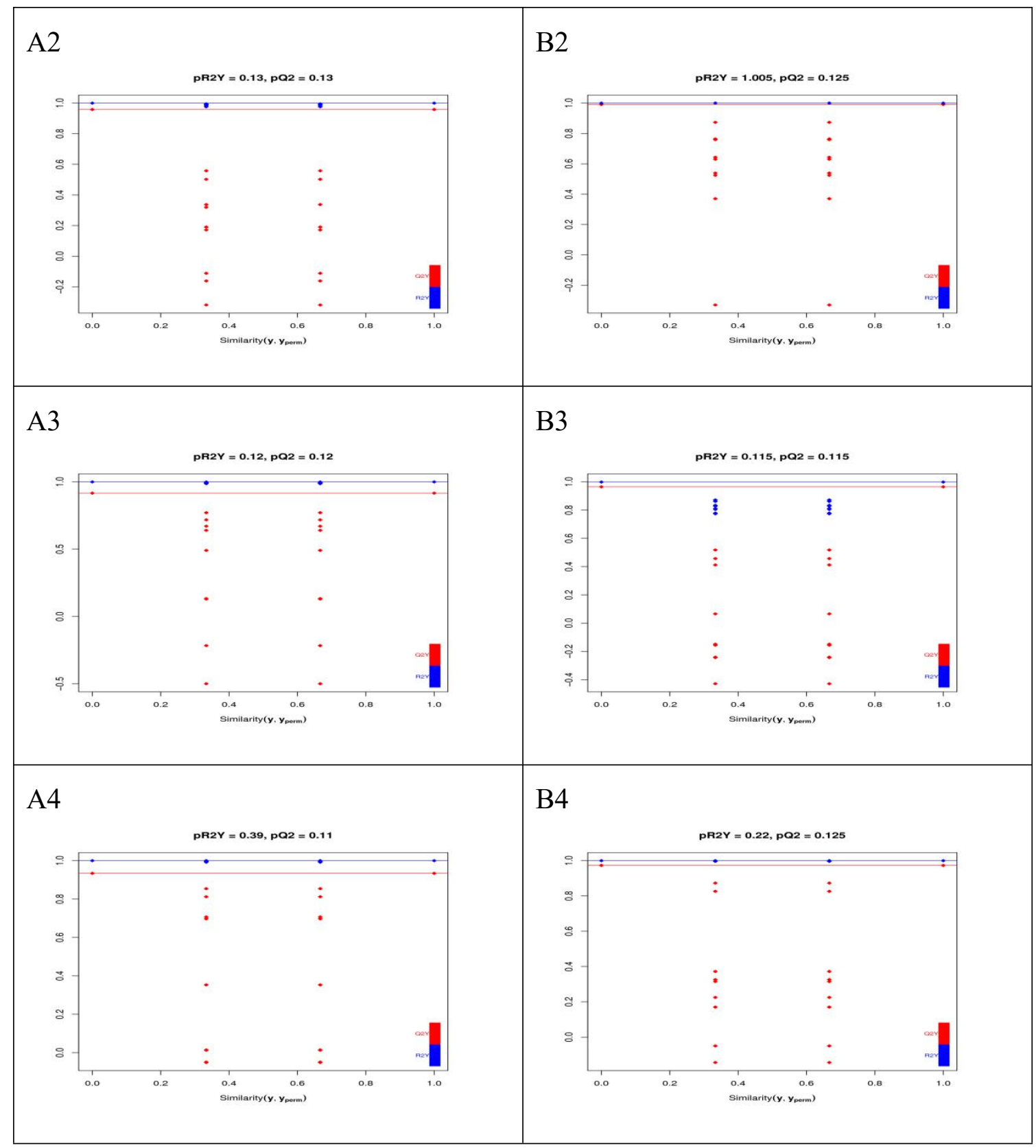

Fig.4 Validation plot of OPLS-DA model. The horizontal line corresponded to R2 and Q2 of the original model, and the blue point and red point represented R2' and Q2' of the model after Y replacement, respectively. (A1)D1 vs D8 in ESI+ mode, (B1) D1 vs D8 in ESI- mode; (A2) D1 vs D15 in ESI+ mode, (B2) D1 vs D15 in ESI- mode; (A3) D1 vs D22 in ESI+ mode, (B3) D1 vs D22 in ESI- mode; (A4) D1 vs D29 in ESI+ mode, (B4) D1 vs D29 in ESI- mode. 


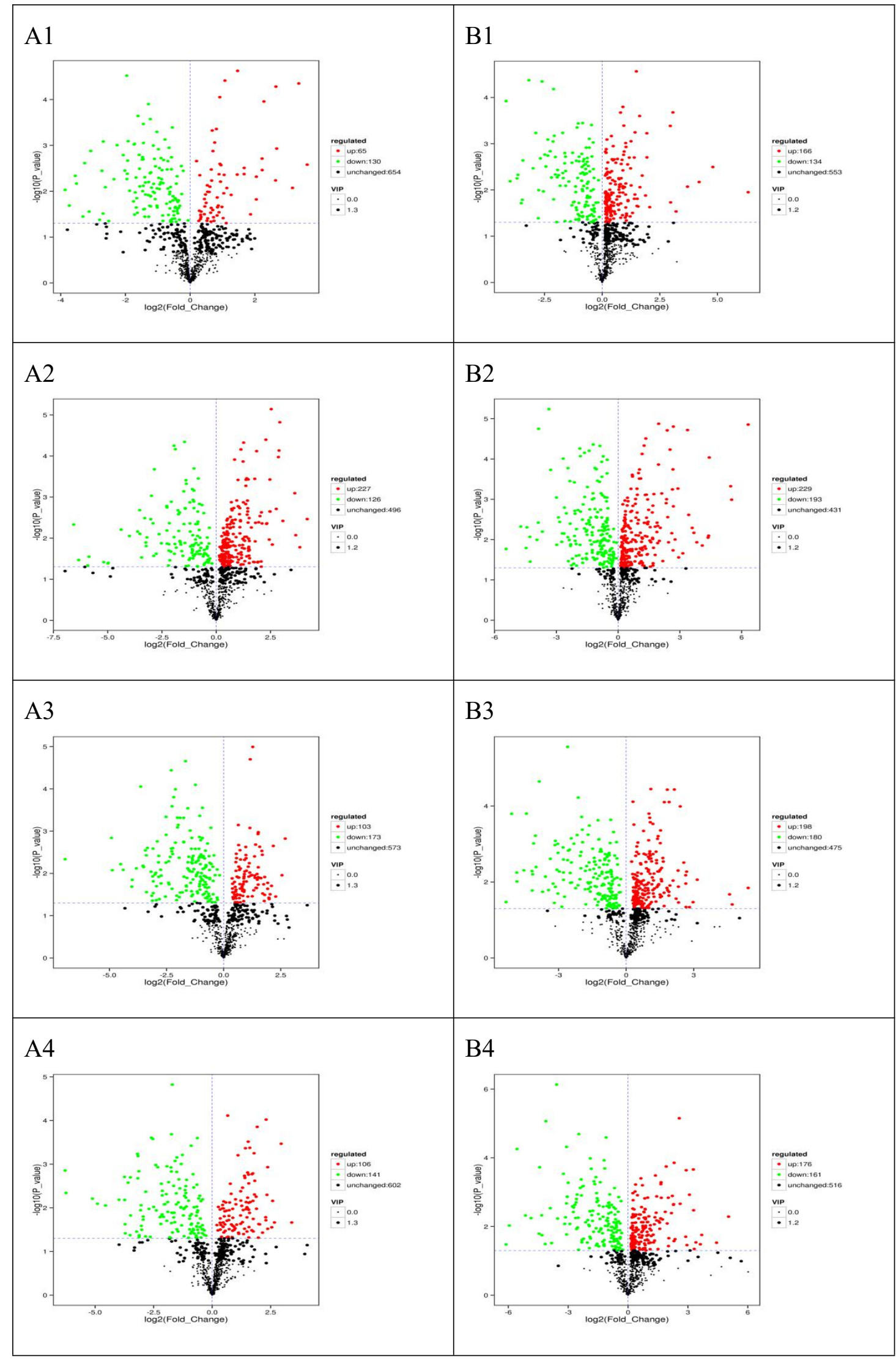

Fig. 5 Volcano Plot with differential metabolites of the four comparison groups. Each point in the figure represents a metabolite, the abscissa represents the log2-fold change of the group compared to the substance, and the ordinate represents the 
$\log 10$-P-value of the student's t-test. The scatter size represents the VIP value of the OPLS-DA model. Green dots represent significantly down-regulated metabolites, red dots represent significantly up-regulated metabolites, and black dots represent metabolites detected but not significantly different. (A1) D1 vs D8 in ESI+ mode, (B1) D1 vs D8 in ESI- mode; (A2) D1 vs D15 in ESI+ mode, (B2) D1 vs D15 in ESImode; (A3) D1 vs D22 in ESI+ mode, (B3) D1 vs D22 in ESI- mode; (A4) D1 vsD29 in ESI+ mode, (B4) D1 vs D29 in ESI- mode.

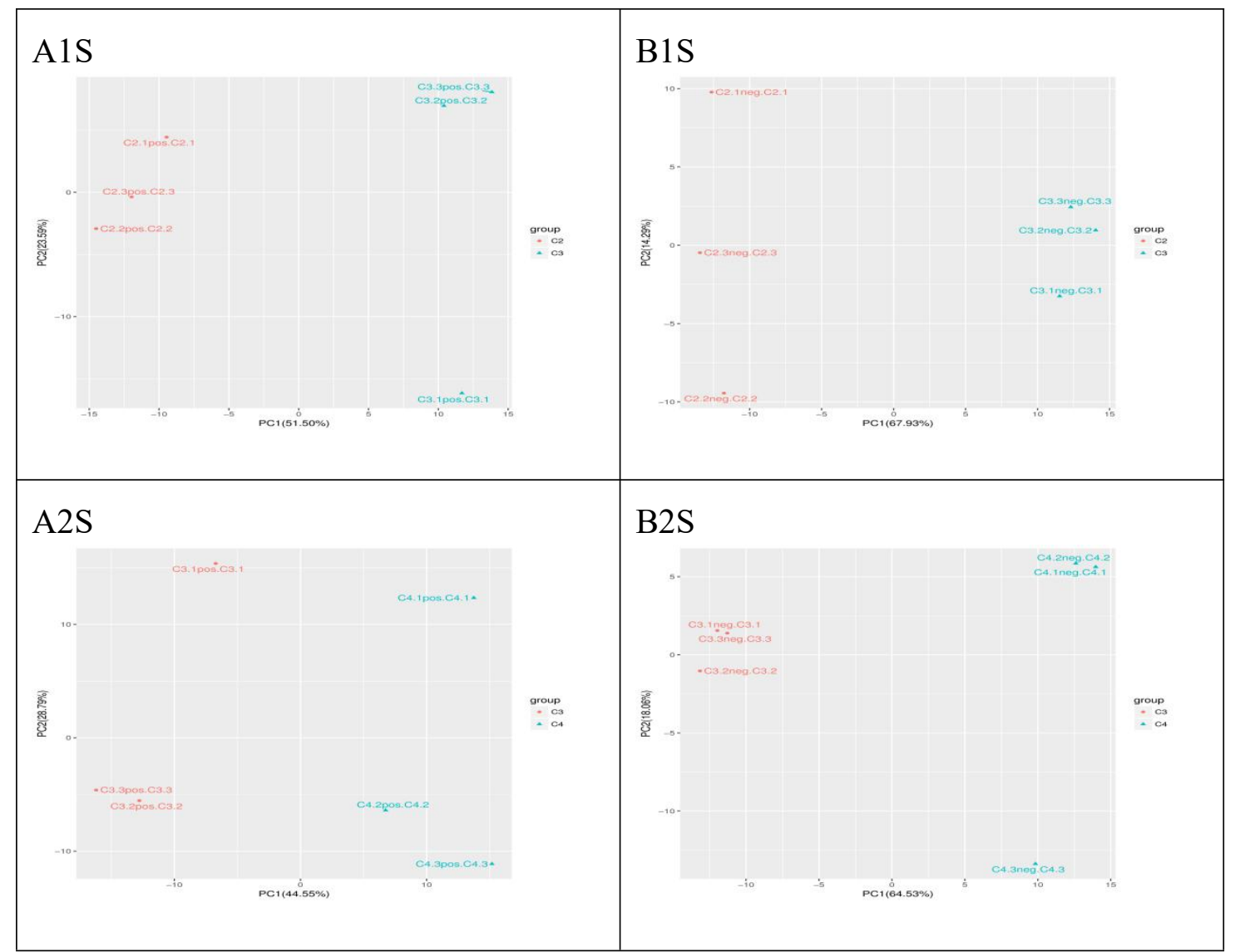

Fig. 2S PCA score plots in D8 vs D15, D15 vs D22 in ESI+ mode (A) and ESI- mode (B). (A1S) D8 vs D15 in ESI+ mode, (B1S) D8 vs D15 in ESI- mode; (A2S) D15 vs D22in ESI+ mode, (B2S) D15 vsD22 in ESI- mode.

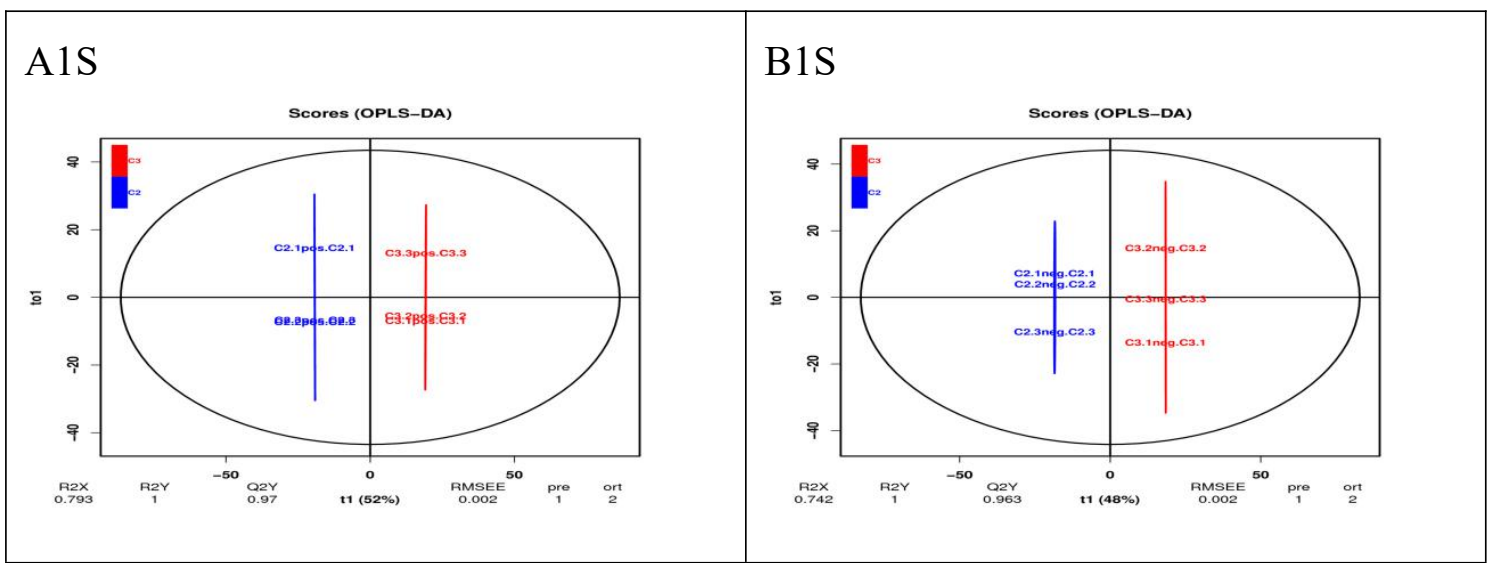




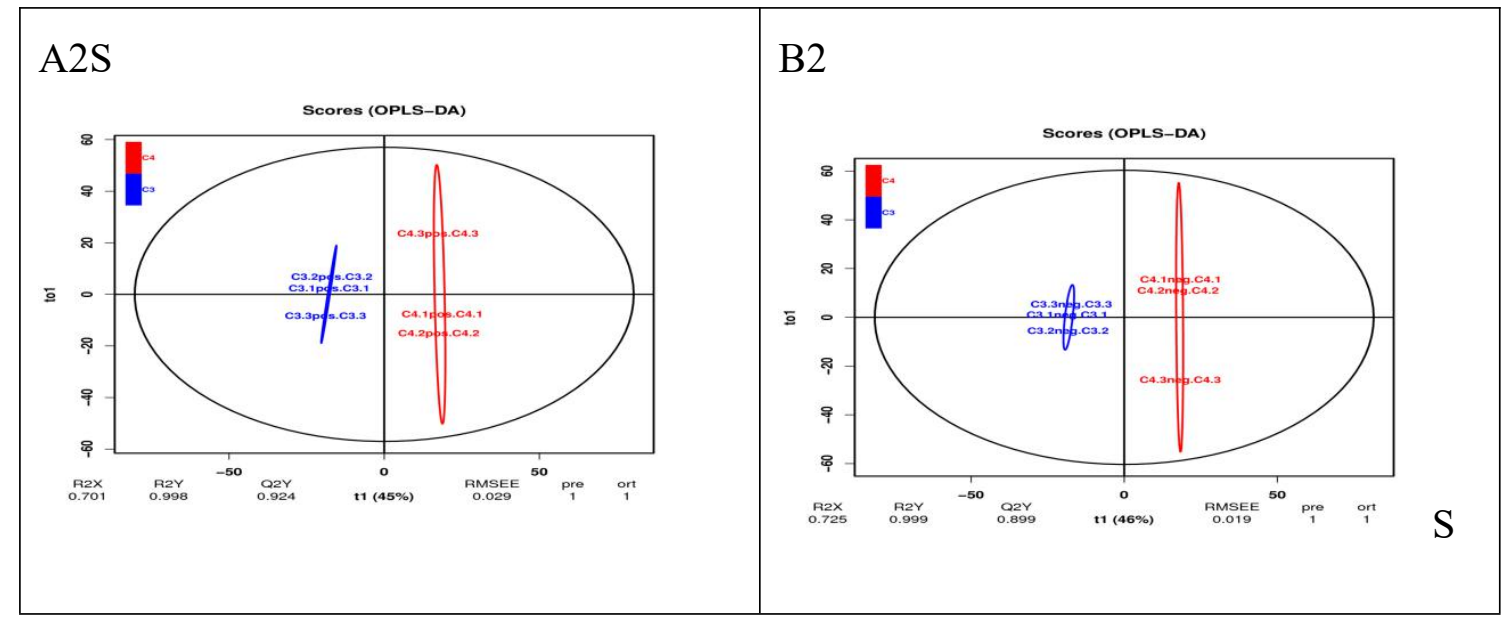

Fig. 3S OPLS-DA score plot D8 vs D15, D15 vsD22 in ESI+ mode (A) and ESImode (B). (A1S) D8 vsD15 in ESI+ mode, (B1S) D8 vs D15 in ESI- mode; (A2S) D15 vs D22 in ESI+ mode, (B2S) D15 vs D22 in ESI- mode.

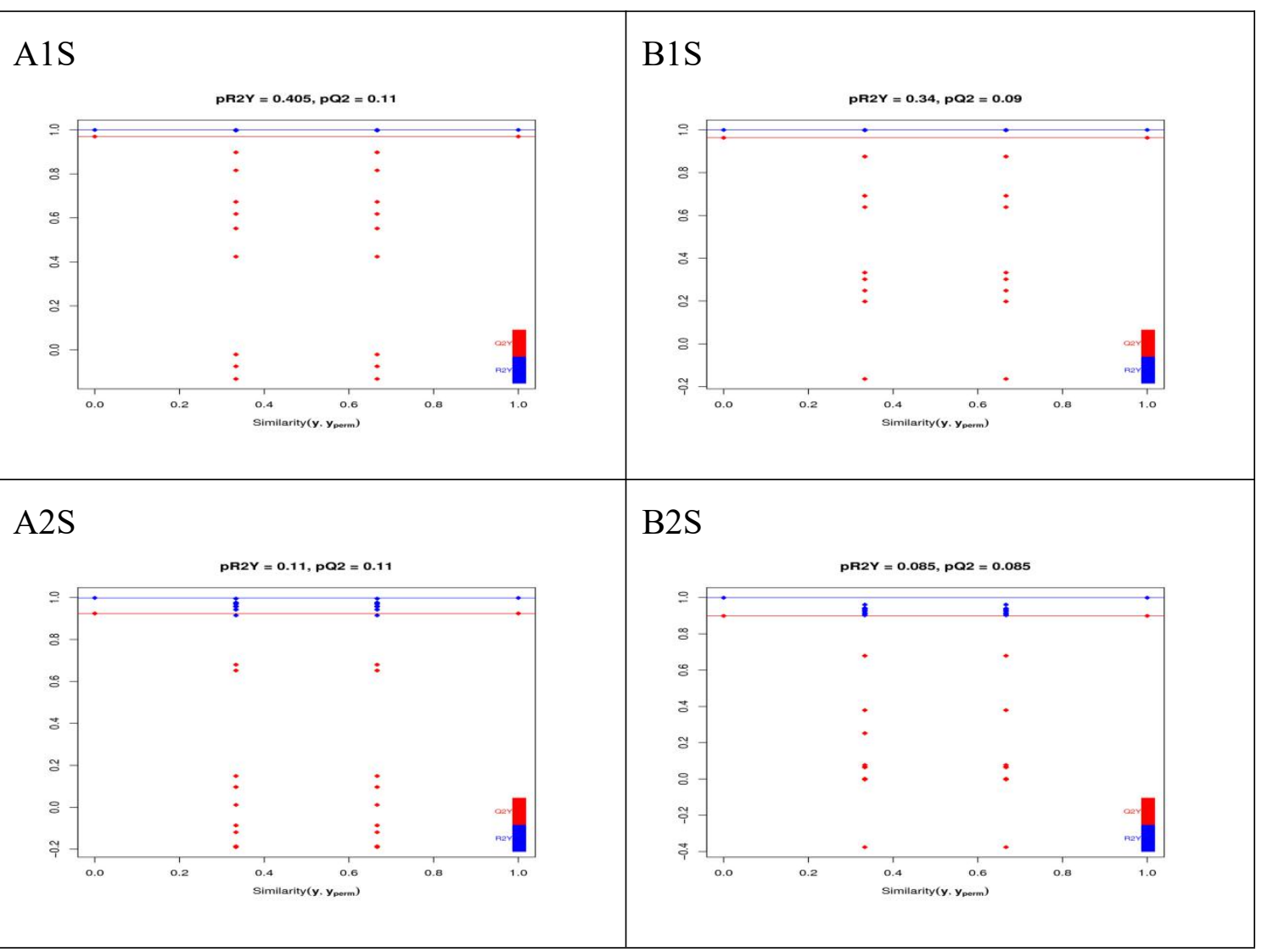

Fig.4S Validation plot of OPLS-DA model D8 vs D15, D15 vsD22in ESI+ mode (A) and ESI- mode (B). The horizontal line corresponded to R2 and Q2 of the original 
554 model, and the blue point and red point represented R2' and Q2' of the model after Y

555 replacement, respectively. (A1S) D8 vs D15 in ESI+ mode, (B1S) D8 vs D15 in ESI-

556 mode; (A2S) D15 vs D22 in ESI+ mode, (B2S) D15 vs D22 in ESI- mode.

557

558

559

560

561

562

563

564 
Figures

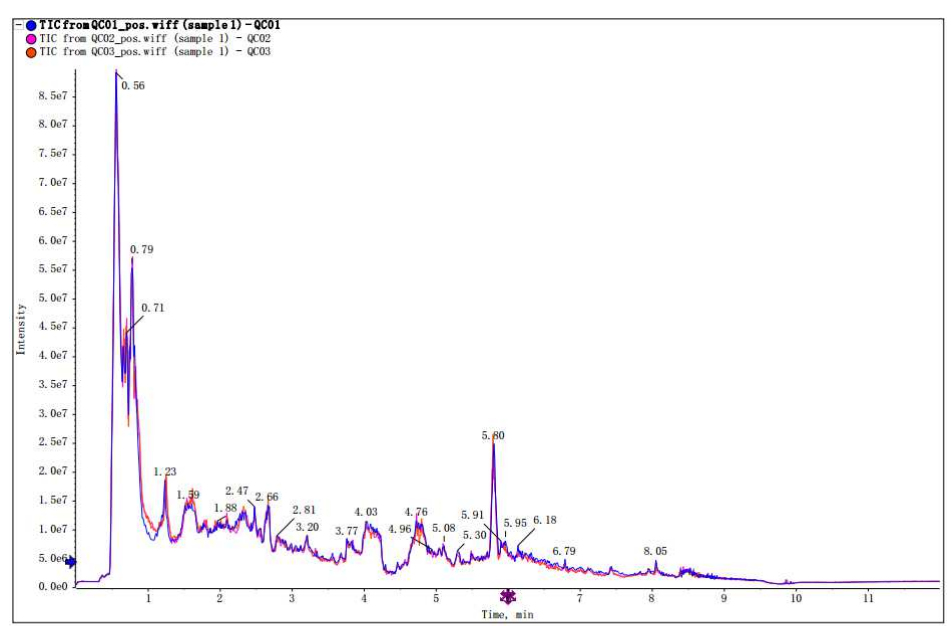

Fig. 1-A

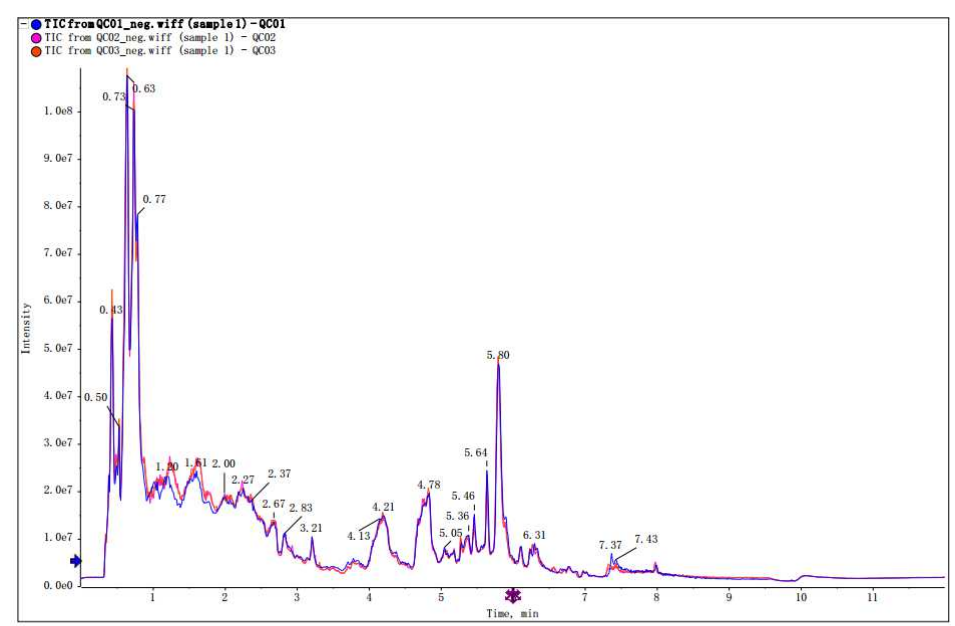

Fig. 1-B

\section{Figure 1}

Total lon Chromatogram of QC in ESI+ mode (A) and ESI -mode (B)

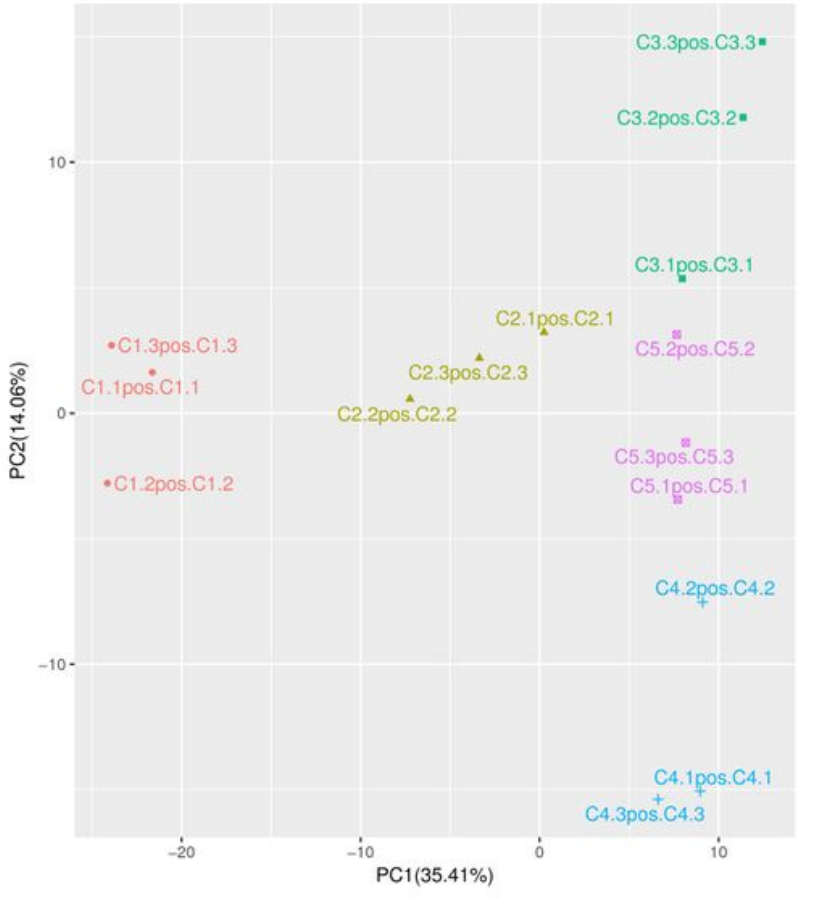

Fig. 2-A

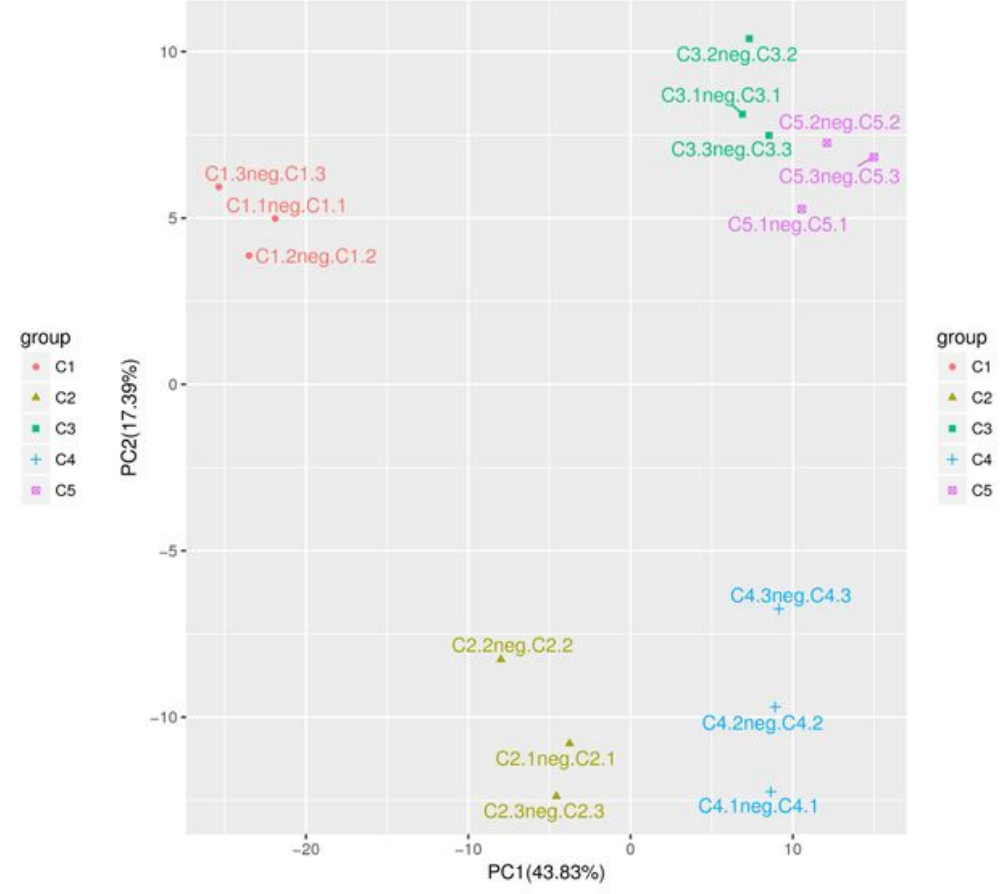

Fig. 2-B

\section{Figure 2}

PCA score plots in the five groups in ESI+ mode (A) and ESI- mode (B). 

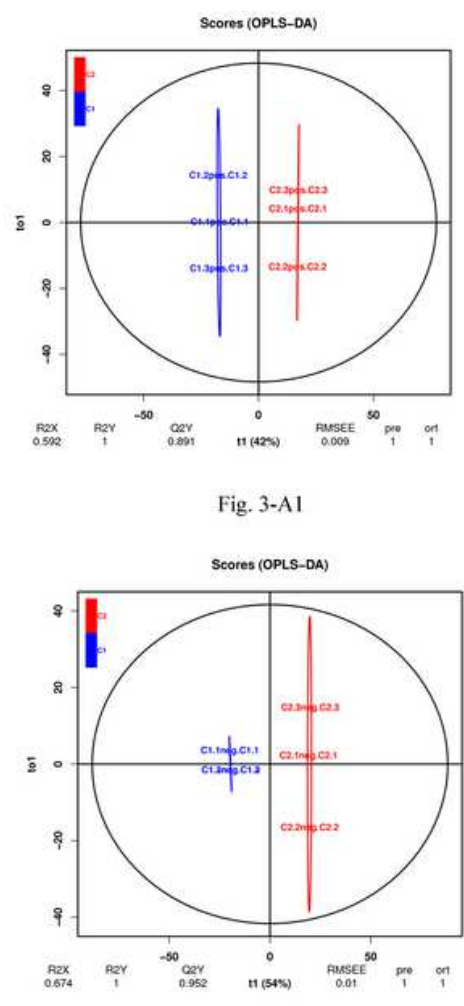

Fig. 3-B1

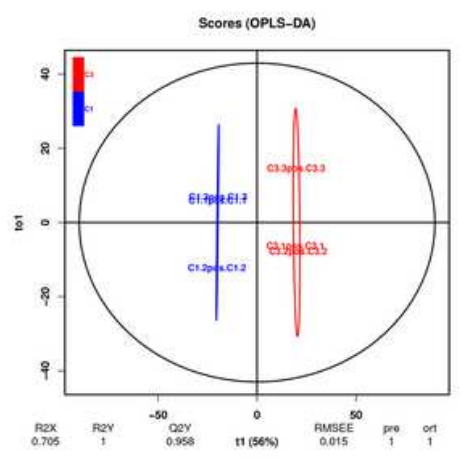

Fig. 3-A2

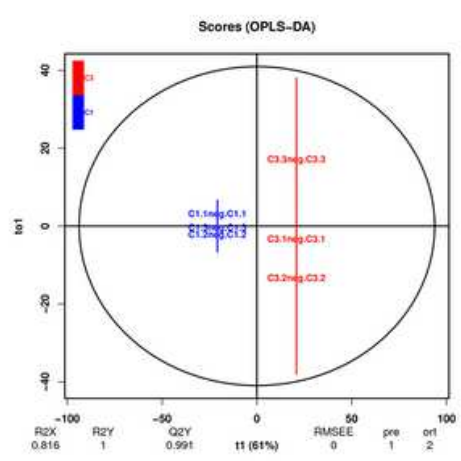

Fig. 3-B2

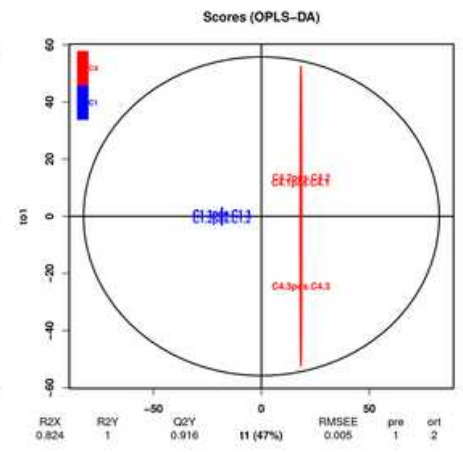

Fig. 3-A3

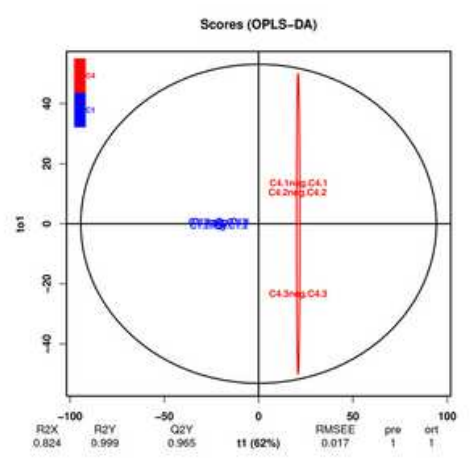

Fig. 3-B3

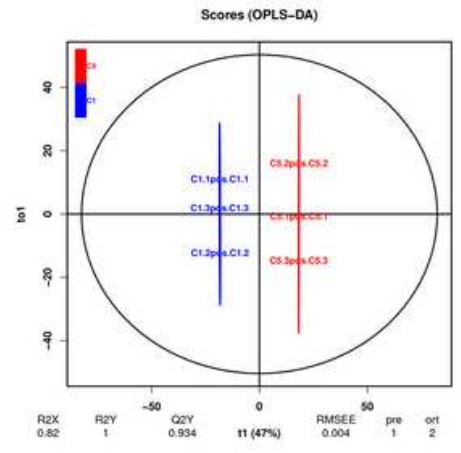

Fig. 3-A4

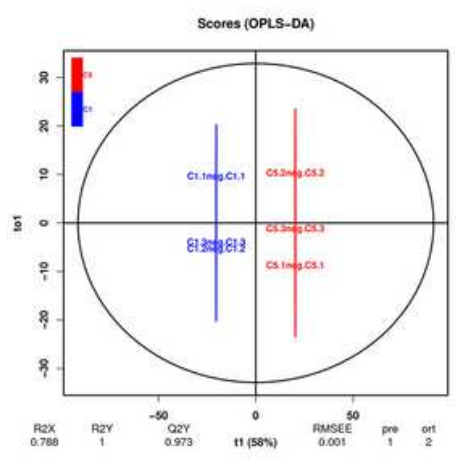

Fig. 3-B4

Figure 3

OPLS-DA score plot of the four comparisons: (A1) D1 vs D8 in ESI+ mode, (B1) D1 vs D8 in ESI- mode; (A2) D1 vs D15 in ESI+ mode, (B2) D1 vs D15 in ESI mode; (A3) D1 vs D22 in ESI+ mode, (B3) D1 vs D22 in ESI- mode; (A4) D1 vs D29 in ESI+ mode, (B4) D1 vs D29 in ESI- mode. 

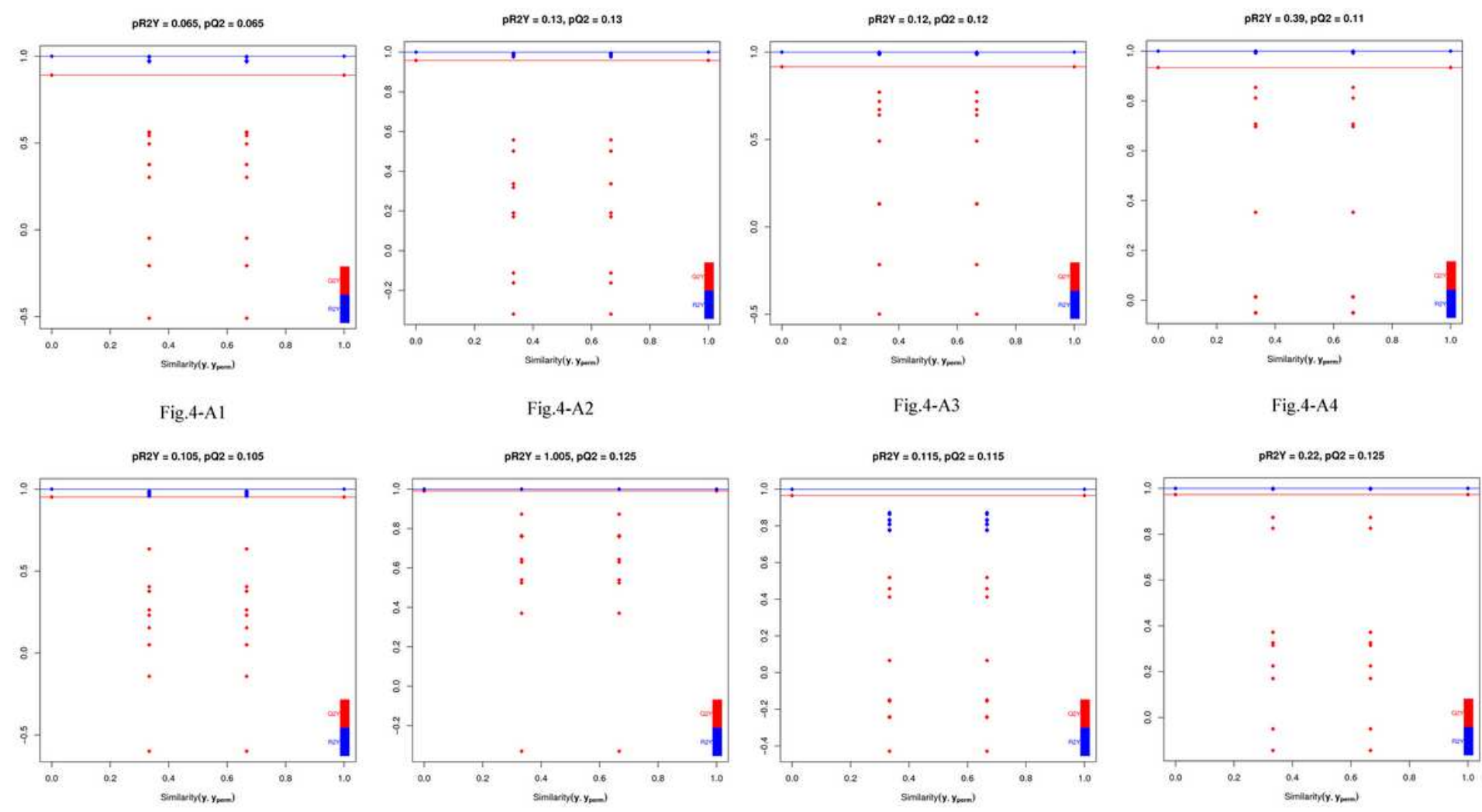

Fig.4-B1

Fig.4-B2

Fig.4-B3

Fig.4-B4

Figure 4

Validation plot of OPLS-DA model. The horizontal line corresponded to R2 and Q2 of the original model, and the blue point and red point represented R2' and Q2' of the model after Y replacement, respectively. (A1)D1 vs D8 in ESI+ mode, (B1) D1 vs D8 in ESI- mode; (A2) D1 vs D15 in ESI+ mode, (B2) D1 vs D15 in ESI- mode; (A3) D1 vs D22 in ESI+ mode, (B3) D1 vs D22 in ESI- mode; (A4) D1 vs D29 in ESI+ mode, (B4) D1 vs D29 in ESI- mode. 


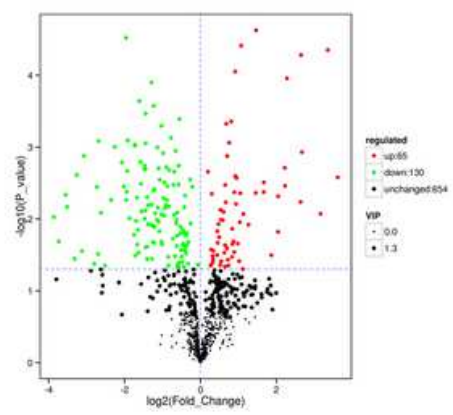

Fig. 5-A1

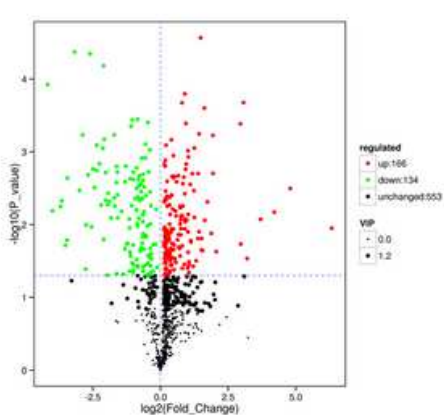

Fig. 5-B1

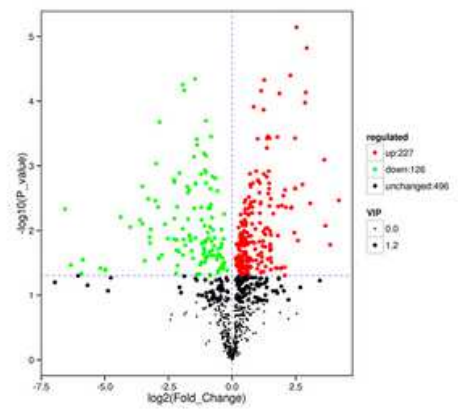

Fig. 5-A2

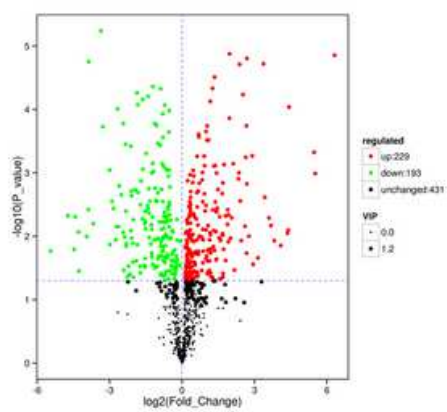

Fig. 5-B2

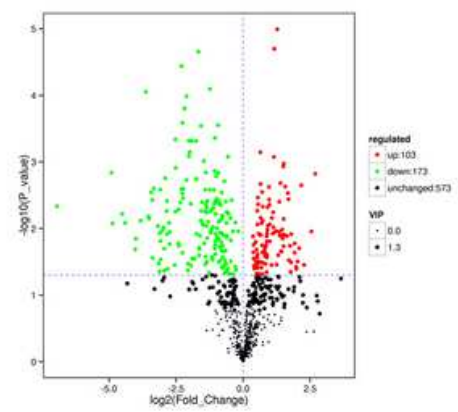

Fig. 5-A3

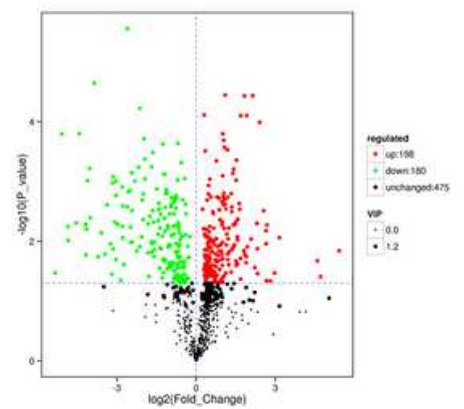

Fig. 5-B3

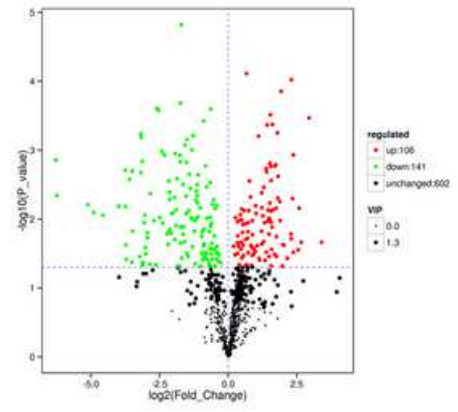

Fig. 5-A4

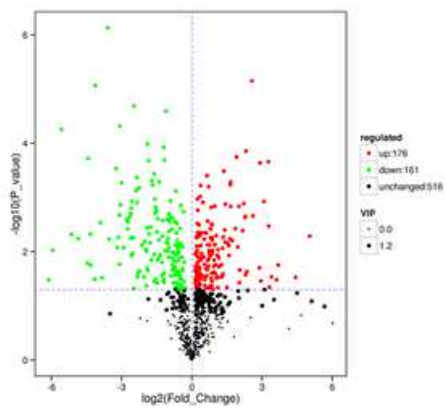

Fig. 5-B4

\section{Figure 5}

Volcano Plot with differential metabolites of the four comparison groups. Each point in the figure represents a metabolite, the abscissa represents the log2-fold change of the group compared to the substance, and the ordinate represents the log10-P-value of the student's t-test. The scatter size represents 536 the VIP value of the OPLS-DA model. Green dots represent significantly down-regulated metabolites, red dots represent significantly up-regulated metabolites, and black dots represent metabolites detected but not significantly different. (A1) D1 vs D8 in ESI+ mode, (B1) D1 vs D8 in ESImode; (A2) D1 vs D15 in ESI+ mode, (B2) D1 vs D15 in ESI mode; (A3) D1 vs D22 in ESI+ mode, (B3) D1 vs D22 in ESI- mode; (A4) D1 vsD29 in ESI+ mode, (B4) D1 vs D29 in ESI- mode.

\section{Supplementary Files}

This is a list of supplementary files associated with this preprint. Click to download.

- supplement4D1vsD15..xls

- QCposTIC.png

- supplement5posD1vsD29.xls

- Fig4SA1A2B1B2.jpg

- Fig2SA1A2B1B2.jpg

- Fig3SA1A2B1B2.jpg

- supplement1POS.xls 
- QCnegTIC.png

- supplement2D1vsD8.xls

- supplement1NEG.xls

- supplement3D1vsD15..xls

- supplement3D1vsD15..xIs

- supplement2NEGD1vsD8.xls

- supplement1POS.xls

- Fig2SA1A2B1B2.jpg

- Fig4SA1A2B1B2.jpg

- supplement5NEGD1vsS29.xls

- supplement5NEGD1vsS29.xls

- supplement4D1vsD15..xls

- Fig3SA1A2B1B2.jpg

- supplement2NEGD1vsD8.xls

- QCnegTIC.png

- supplement2D1vsD8.xls

- supplement1NEG.xls

- QCposTIC.png

- supplement5posD1vsD29.xls 